J. Korean Math. Soc. 50 (2013), No. 6, pp. 1349-1367

http://dx.doi.org/10.4134/JKMS.2013.50.6.1349

\title{
WEIGHTED MOORE-PENROSE INVERTIBLE AND WEIGHTED EP BANACH ALGEBRA ELEMENTS
}

\author{
Enrico Boasso, Dragan S. Djordjević, and Dijana Mosić
}

Abstract. The weighted Moore-Penrose inverse will be introduced and studied in the context of Banach algebras. In addition, weighted EP Banach algebra elements will be characterized. The Banach space operator case will be also considered.

\section{Introduction}

The Moore-Penrose inverse in Banach algebras was introduced by V. Rakočević in [22]. Some of its basic properties were studied in this paper, and later in [23], [16] and [1]. In the recent past, a particular class of Moore-Penrose invertible objects were studied, namely, EP Banach space operators and EP Banach algebra elements, in other words, Moore-Penrose invertible operators or elements of an algebra such that they commute with their Moore-Penrose inverse (see $[1,2,3,17]$ ). It is worth noting that EP objects have been intensively studied in different contexts such as matrices, Hilbert space bounded and linear maps, $C^{*}$-algebras, rings with involution and naturally in the two mentioned above. Moreover, they consist in a generalization of the notion normal and hermitian objects (see [3, Theorems 3.1 and 3.3]).

In the recent work by Y. Tian and H. Wang [24], the concept of weighted EP matrices (matrices that commute with their weighted Moore-Penrose inverse) was introduced. What is more, the second and third authors studied weighted EP $C^{*}$-algebra elements in $[18,19]$. The main objective of this paper is to introduce and study similar objects in the contexts of Banach space operators and Banach algebra elements.

In fact, in Section 2, after having recalled some basic definitions and results, the notion of weighted Moore-Penrose invertible Banach space operators

Received February 2, 2013.

2010 Mathematics Subject Classification. Primary 46H05; Secondary 15A09.

Key words and phrases. (weighted) Moore-Penrose inverse, (weighted) EP element, group inverse, Banach algebra, Banach space operator.

The second and third authors are supported by the Ministry of Science, Republic of Serbia, grant no. 174007 . 
and Banach algebra elements is introduced and studied. Furthermore, in Section 3, weighted EP Banach space operators and Banach algebra elements are characterized using the concept of group inverse. It is worth noting that the results presented in this paper apply to arbitrary Banach algebras, operators on Banach spaces and matrices with any norm.

\section{The weighted Moore-Penrose inverse}

From now on, $X$ will denote a Banach space and $L(X)$ the Banach algebra of all bounded and linear maps defined on and with values in $X$. If $T \in L(X)$, then $N(T)$ and $R(T)$ will stand for the null space and the range of $T$, respectively. In addition, $X^{*}$ will denote the dual space of $X$ and if $T \in L(X)$, then $T^{*} \in L\left(X^{*}\right)$ will stand for the adjoint map of $T \in L(X)$.

On the other hand, $A$ will denote a complex unital Banach algebra with unit 1. In addition, the set of all invertible elements of $A$ will be denoted by $A^{-1}$. If $a \in A$, then $L_{a}: A \rightarrow A$ and $R_{a}: A \rightarrow A$ will denote the map defined by left and right multiplications, respectively:

$$
L_{a}(x)=a x, R_{a}(x)=x a,
$$

where $x \in A$. Moreover, the following notations will be used: $N\left(L_{a}\right)=a^{-1}(0)$ and $R\left(L_{a}\right)=a A$.

Recall that an element $a \in A$ is called regular if it has a generalized inverse, namely, if there exists $b \in A$ such that

$$
a=a b a .
$$

Furthermore, a generalized inverse $b$ of a regular element $a \in A$ will be called normalized if $b$ is regular and $a$ is a generalized inverse of $b$, equivalently,

$$
a=a b a, b=b a b .
$$

Next follows the key notion in the definition of the (weighted) Moore-Penrose inverse in the context of Banach algebras.

Definition 2.1. Given a unital Banach algebra $A$, an element $a \in A$ is said to be hermitian if $\|\exp (i t a)\|=1$ for all $t \in \mathbb{R}$.

In regards to equivalent definitions and main properties of hermitian Banach algebra elements and Banach space operators, see, for example, [4, 7, 15, 20, 25]. Recall that if $A$ is a $C^{*}$-algebra, then $a \in A$ is hermitian if and only if $a$ is self-adjoint (see [4, Proposition 20, Chapter I, Section 12]). Given $A$ a unital Banach algebra, the set of all Hermitian elements of $A$ will be denoted by $H(A)$.

Now the notion of Moore-Penrose invertible Banach algebra element will be recalled.

Definition 2.2. Let $A$ be a unital Banach algebra and $a \in A$. If there exists $x \in A$ such that $x$ is a normalized generalized inverse of $a$ and $a x$ and $x a$ are hermitian, then $x$ is said to be the Moore-Penrose inverse of $a$, and is denoted by $a^{\dagger}$. 
Recall that according to [22, Lemma 2.1], there is at most one Moore-Penrose inverse. Concerning the Moore-Penrose inverse in Banach algebras, see $[1,2$, $3,16,17,22,23]$. In the context of $C^{*}$-algebras, see $[9,10,13]$. For the original definition of the Moore-Penrose inverse for matrices, see [21].

Next the weighted Moore-Penrose inverse will be considered. Recall that given a $C^{*}$-algebra $A$ and $a \in A, b \in A$ is said to be the weighted MoorePenrose inverse of $a$ with weights $e$ and $f$ if the following identities hold:

$$
a b a=a, b a b=b,(e a b)^{*}=e a b,(f b a)^{*}=f b a,
$$

where $e$ and $f$ are positive and invertible elements in $A$ (see $[14,18,19]$ ).

According to [14], the conditions defining the weighted Moore-Penrose inverse can be rewritten as

$$
a b a=a, b a b=b,(a b)^{* e}=a b,(b a)^{* f}=b a,
$$

where $A^{* e}=\left(A^{* e},\|\cdot\|_{e}\right)$ (respectively, $\left.A^{* f}=\left(A^{* f},\|\cdot\|_{f}\right)\right)$ is the $C^{*}$-algebra with underlying space $A$, involution $x \rightarrow x^{* e}=e^{-1} x^{*} e$ (respectively, $x \rightarrow$ $x^{* f}=f^{-1} x^{*} f$ ) and norm $\|x\|_{e}=\left\|e^{1 / 2} x e^{-1 / 2}\right\|$ (respectively, $\|x\|_{f}=\|$ $\left.f^{1 / 2} x f^{-1 / 2} \|\right), x \in A$. Next weighted Moore-Penrose invertible Banach algebra elements will be introduced. To this end, however, some preparation in needed.

Let $A$ be a complex unital Banach algebra and consider $a \in A$. The element $a$ is said to be positiveif $V(a) \subset \mathbb{R}_{+}$, where $V(a)=\left\{f(a): f \in A^{*},\|f\| \leq 1\right.$, $f(1)=1\}\left(\left[4\right.\right.$, Definition 5, Chapter V, Section 38]). Denote by $A_{+}$the set of all positive elements of $A$. Note that the necessary and sufficient condition for $a \in A$ to be positive is that $a$ is hermitian and $\sigma(a) \subset \mathbb{R}_{+}$([4, Definition 5, Chapter V, Section 38]). Recall that according to [4, Lemma 7, Chapter V, Section 38], if $c \in A_{+}$, then there exists $d \in A_{+}$such that $d^{2}=c$. Moreover, according to [8, Theorem], the square root is unique. In particular, the square root of $c$ will be denoted by $c^{1 / 2}$. For the definition and equivalent conditions of positive $C^{*}$-algebra elements, see [5, Definition 3.1 and Theorem 3.6, Chapter VIII, Section 3].

Given a complex unital Banach algebra $A$ and $u \in A^{-1} \cap A_{+}$, denote by $A^{u}=\left(A^{u},\|\cdot\|_{u}\right)$ the complex unital Banach algebra with underlying space $A$ and norm $\|x\|_{u}=\left\|u^{1 / 2} x u^{-1 / 2}\right\|$. When $A$ is a $C^{*}$-algebra, according to [4, Proposition 20, Chapter I, Section 12], $a$ is self-adjoint in $\left(A^{* u},\|\cdot\|_{u}\right)$ if and only if $a$ is hermitian in $\left(A^{u},\|\cdot\|_{u}\right)$, where if $x \in A$, then as before, the involution in $A^{* u}$ is defined as follows: $x \rightarrow x^{* u}=u^{-1} x^{*} u$. These facts lead to the following definition.

Definition 2.3. Let $A$ be a complex unital Banach algebra and consider $e$ and $f$ two positive invertible elements in $A$. The element $a \in A$ is said to be weighted Moore-Penrose invertible with weights $e$ and $f$ if there exists $b \in A$ such that $b$ is a normalized generalized inverse of $a$ and $a b$ (respectively, $b a$ ) is a hermitian element of $A^{e}$ (respectively, of $A^{f}$ ). 
Clearly, the conditions in Definition 2.3 extend the notion of a weighted Moore-Penrose invertible $C^{*}$-algebra element to Banach algebras ([14]). Furthermore, note that if $e=f$, then the necessary and sufficient condition for $a \in A$ to be weighted Moore-Penrose invertible with weight $e$ is that $a \in A^{e}$ (respectively, $A^{* e}$ when $A$ is a $C^{*}$-algebra) is Moore-Penrose invertible. In particular, when both weights coincide, the weighted Moore-Penrose inverse reduces to the Moore-Penrose inverse, naturally changing the structure of the Banach or $C^{*}$-algebra. In what follows, some basic properties of weighted Moore-Penrose invertible Banach algebra elements will be studied.

Firstly, the uniqueness of the weighted Moore-Penrose inverse will be proved. Moreover, ideas similar to those in [22, Lemma 2.1] will be used. However, before considering the mentioned property, some preparation is needed.

Lemma 2.4. Let $A$ be a unital Banach algebra and consider $u \in A^{-1} \cap A_{+}$. Then $L_{u} \in L(A)$ is invertible and positive.

Proof. It is clear that $L_{u} \in L(A)$ is invertible. In addition, note that since $A$ is unital, $\left\|L_{u}\right\|=\|u\|$. In particular, since

$$
\left\|\exp \left(i t L_{u}\right)\right\|=\left\|L_{\exp (i t u)}\right\|=\|\exp (i t u)\|=1,
$$

$L_{u} \in H(L(A))$. Moreover, since $\sigma\left(L_{u}\right)=\sigma(u)$ by [4, Proposition 4, Chapter I, Section 5], $L_{u} \in L(A)_{+}([4$, Definition 5, Chapter V, Section 38]).

Proposition 2.5. Let $A$ be a complex unital Banach algebra and consider e, $f \in A^{-1} \cap A_{+}$. Then for $a \in A$, there is at most one weighted Moore-Penrose inverse of a with weights $e$ and $f$.

Proof. Suppose that $b$ and $c$ are two weighted Moore-Penrose inverses of $a$ with weights $e$ and $f$ and consider $L_{a b}, L_{a c} \in L(A)^{L_{e}}$. A straightforward calculation proves that

$$
\left\|\exp \left(i t L_{a b}\right)\right\|_{L_{e}}=\|\exp (i t a b)\|_{e}=1,\left\|\exp \left(i t L_{a c}\right)\right\|_{L_{e}}=\|\exp (i t a c)\|_{e}=1,
$$

equivalently, $L_{a b}$ and $L_{a c}$ are two hermitian idempotents of $L(A)^{L_{e}}$. Moreover, since $b$ and $c$ are two normalized generalized inverses of $a$, it is not difficult to prove that $R\left(L_{a b}\right)=R\left(L_{a}\right)=R\left(L_{a c}\right)$. Therefore, according to [20, Theorem 2.2], $L_{a b}=L_{a c}$. In particular, $a b=a c$.

A similar argument, using in particular $R_{b a}$ and $R_{c a}$ instead of $L_{a b}$ and $L_{a c}$, respectively, proves that $b a=c a$. Then

$$
b=b a b=c a b=c a c=c .
$$

According to Proposition 2.5, given a complex unital Banach algebra and $a \in A$, if the weighted Moore-Penrose inverse of $a$ exists, then it will be denoted by $a_{e, f}^{\dagger}$. In the following remarks, some elementary facts that will be used in this paper will be presented. 
Remark 2.6. (a) Let $A$ be a complex unital Banach algebra and consider $e$, $f \in A^{-1} \cap A_{+}$. Suppose that $a_{e, f}^{\dagger}$ exists, $a \in A$. Then the following statements can be easily derived from the conditions characterizing the weighted MoorePenrose inverse of $a$.

(i) $\left(a_{e, f}^{\dagger}\right)_{f, e}^{\dagger}=a$.

(ii) $a_{e, f}^{\dagger} A=a_{e, f}^{\dagger} a A, a a_{e, f}^{\dagger} A=a A$.

(iii) $\left(a a_{e, f}^{\dagger}\right)^{-1}(0)=\left(a_{e, f}^{\dagger}\right)^{-1}(0),\left(a_{e, f}^{\dagger} a\right)^{-1}(0)=a^{-1}(0)$.

(iv) $A=a_{e, f}^{\dagger} A \oplus a^{-1}(0)=a A \oplus\left(a_{e, f}^{\dagger}\right)^{-1}(0)$.

(b) Suppose that $A=L(X), X$ a Banach space. Let $E, F \in L(X)$ be two invertible positive operators and consider $T \in L(X)$ such that $T_{E, F}^{\dagger}$ exists. Then it is not difficult to prove the following facts.

(v) $R\left(T_{E, F}^{\dagger} T\right)=R\left(T_{E, F}^{\dagger}\right), R\left(T T_{E, F}^{\dagger}\right)=R(T)$.

(vi) $N\left(T_{E, F}^{\dagger} T\right)=N(T), N\left(T T_{E, F}^{\dagger}\right)=N\left(T_{E, F}^{\dagger}\right)$.

(vii) $X=R\left(T_{E, F}^{\dagger}\right) \oplus N(T)=R(T) \oplus N\left(T_{E, F}^{\dagger}\right)$.

Next, conditions equivalent to the existence of the weighted Moore-Penrose inverse will be given. Firstly, the case $A=L(X), X$ a Banach space, will be considered.

Theorem 2.7. Let $X$ be a Banach space and consider $E, F \in L(X)$ two invertible positive operators. Then for $T \in L(X)$, the following statements are equivalent:

(i) $T_{E, F}^{\dagger}$ exists;

(ii) there exist two idempotents $P, Q \in L(X)$ such that $P \in H\left(L(X)^{E}\right)$, $R(P)=R(T)$, and $Q \in H\left(L(X)^{F}\right), N(Q)=N(T)$.

Furthermore, if such $P$ and $Q$ exist, then they are unique.

Proof. If $T_{E, F}^{\dagger}$ exists, then consider $P=T T_{E, F}^{\dagger}$ and $Q=T_{E, F}^{\dagger} T$.

On the other hand, suppose that statement (ii) holds. Consider the invertible operator $T^{\prime} \in L(R(Q), R(T))$,

$$
T^{\prime}=\left.T\right|_{R(Q)} ^{R(T)}: R(Q) \rightarrow R(T),
$$

and define $S \in L(X)$ as follows:

$$
\left.S\right|_{N(P)} \equiv 0,\left.S\right|_{R(T)} ^{R(Q)}=\left(T^{\prime}\right)^{-1} \in L(R(T), R(Q)) .
$$

Since $R(P)=R(T)$, an easy calculation proves that $S$ is a normalized generalized inverse of $T$. Moreover, since $T S$ and $P$ are idempotents of $L(X)$ such that

$$
R(T S)=R(T)=R(P), N(T S)=N(S)=N(P),
$$

it is clear that $T S=P$. In particular, $T S \in H\left(L(X)^{E}\right)$. A similar argument proves that $S T \in H\left(L(X)^{F}\right)$. Consequently, $S=T_{E, F}^{\dagger}$. 
Now suppose that $P^{\prime}$ and $Q^{\prime}$ are two idempotents that satisfy statement (ii). Then $R(P)=R\left(P^{\prime}\right)$ and $R(I-Q)=R\left(I-Q^{\prime}\right)$. Then, according to [25, Hilfssatz 2(a)-(b)] and [20, Theorem 2.2], $P=P^{\prime}$ and $Q=Q^{\prime}$.

In the following theorem, weighted Moore-Penrose invertible Banach algebra elements will be characterized.

Theorem 2.8. Let $A$ be a complex unital Banach algebra and consider $e$, $f \in A^{-1} \cap A_{+}$. If $a$ and $b \in A$ are such that $b$ is a normalized generalized inverse of $a$, then the following statements are equivalent.

(i) $b=a_{e, f}^{\dagger}$;

(ii) $L_{b}=\left(L_{a}\right)_{L_{e}, L_{f}}^{\dagger} \in L(A)$.

In particular, if statements (i)-(ii) hold, then $\left(L_{a}\right)_{L_{e}, L_{f}}^{\dagger}=L_{a_{e, f}^{\dagger}}$.

Proof. First of all, recall that $L_{e}$ and $L_{f} \in L(A)$ are invertible and positive (Lemma 2.4).

Clearly, $b$ is a normalized generalized inverse of $a$ in $A$ if and only if $L_{b}$ is a normalized generalized inverse of $L_{a}$ in $L(A)$. In addition, according to [8, Theorem], $\left(L_{e}\right)^{1 / 2}=L_{e^{1 / 2}}$ and $\left(L_{f}\right)^{1 / 2}=L_{f^{1 / 2}}$ (Lemma 2.4). Furthermore, since

$$
\left\|\exp \left(i t L_{a} L_{b}\right)\right\|_{L_{e}}=\left\|\exp \left(i t L_{a b}\right)\right\|_{L_{e}}=\left\|L_{\exp (i t a b)}\right\|_{L_{e}}=\|\exp (i t a b)\|_{e}
$$

and

$$
\left\|\exp \left(i t L_{b} L_{a}\right)\right\|_{L_{f}}=\left\|\exp \left(i t L_{b a}\right)\right\|_{L_{f}}=\left\|L_{\exp (i t b a)}\right\|_{L_{f}}=\|\exp (i t b a)\|_{f},
$$

$a b \in H\left(A^{e}\right)$ and $b a \in H\left(A^{f}\right)$ if and only if $L_{a} L_{b} \in H\left(L(A)^{L_{e}}\right)$ and $L_{b} L_{a} \in$ $H\left(L(A)^{L_{f}}\right)$. Therefore, statements (i) and (ii) are equivalent.

In the following proposition, the weighted Moore-Penrose inverse will be described in a particular case. Recall that according to [9, Thorem 6], the condition of being regular is equivalent to that of being Moore-Penrose invertible for $C^{*}$-algebra elements. However, according to [1, Remark 4], in a general Banach algebra, these two notions are not in general equivalent. Compare the next proposition with [14, Theorem 5].

Proposition 2.9. Let $A$ be a complex unital Banach algebra and consider $e$, $f \in A^{-1} \cap A_{+}$. Then for $a \in A$ such that $e^{1 / 2} a f^{-1 / 2} \in A$ is Moore-Penrose invertible,

$$
a_{e, f}^{\dagger}=f^{-1 / 2}\left(e^{1 / 2} a f^{-1 / 2}\right)^{\dagger} e^{1 / 2} .
$$

Proof. Let $c=e^{1 / 2} a f^{-1 / 2}$ and $b=f^{-1 / 2} c^{\dagger} e^{1 / 2}$. Then, using in particular that $a=e^{-1 / 2} c f^{1 / 2}$, it is not difficult to prove that $a b a=a, b a b=b, a b=$ $e^{-1 / 2} c c^{\dagger} e^{1 / 2}$ and $b a=f^{-1 / 2} c^{\dagger} c f^{1 / 2}$. However, since $c c^{\dagger}$ and $c^{\dagger} c$ are hermitian elements of $A, a b \in H\left(A^{e}\right)$ and $b a \in H\left(A^{f}\right)$. 
Next, weighted Moore-Penrose inverses in quotient algebras will be considered. First of all, given a complex unital Banach algebra $A$ and $J$ a proper closed two-sided ideal of $A$, the quotient map is denoted by $\Pi: A \rightarrow A / J$. In addition, if $a \in A$, then the quotient class of $a$ is denoted by $\tilde{a}=\Pi(a)$.

Lemma 2.10. Let $A$ be a complex unital Banach algebra and consider $J \subset A$ a proper closed two-sided ideal. Then for $u \in A^{-1} \cap A_{+}, \tilde{u}$ is invertible and positive in $A / J$.

Proof. Clearly, $\tilde{u} \in A / J$ is invertible. In addition, if $\|\cdot\|^{\prime}$ denotes the norm in $A / J$, then

$$
\|\exp (i t \tilde{u})\|^{\prime}=\|\Pi(\exp (i t u))\|^{\prime} \leq\|\exp (i t u)\|=1 .
$$

Consequently, according to $[1, \operatorname{Remark} 2], \tilde{u} \in H(A / J)$. Furthermore, since, according to [4, Lemma 7, Chapter V, Section 38], there exists $v \in A_{+}$such that $v^{2}=u, \tilde{v}^{2}=\tilde{u}$ and $\sigma(\tilde{u}) \subset \mathbb{R}_{+}$. Therefore, according to [4, Definition 5 , Chapter V, Section 38], $\tilde{u} \in(A / J)_{+}$.

Theorem 2.11. Let $A$ be a complex unital Banach algebra and consider e, $f \in A^{-1} \cap A_{+}$. If $a_{e, f}^{\dagger}$ exists, then $\tilde{a} \in A / J$ is weighted Moore-Penrose invertible with weights $\tilde{e}$ and $\tilde{f}$. Furthermore, $\tilde{a}_{\tilde{e}, \tilde{f}}^{\dagger}=\Pi\left(a_{e, f}^{\dagger}\right)$.

Proof. It is clear that $\Pi\left(a_{e, f}^{\dagger}\right)$ is a normalized generalized inverse of $\tilde{a}$. On the other hand, note that according to [8, Theorem], $\Pi\left(e^{1 / 2}\right)=\tilde{e}^{1 / 2}, \Pi\left(e^{-1 / 2}\right)=$ $\tilde{e}^{-1 / 2}, \Pi\left(f^{1 / 2}\right)=\tilde{f}^{1 / 2}$ and $\Pi\left(f^{-1 / 2}\right)=\tilde{f}^{-1 / 2}$. As a result,

$$
\begin{aligned}
\left\|\exp \left(i t \tilde{a} \Pi\left(a_{e, f}^{\dagger}\right)\right)\right\|_{\tilde{e}}^{\prime} & =\left\|\exp \left(i t \Pi\left(a a_{e, f}^{\dagger}\right)\right)\right\|_{\tilde{e}}^{\prime}=\left\|\Pi\left(e^{1 / 2} \exp \left(i t a a_{e, f}^{\dagger}\right) e^{-1 / 2}\right)\right\|^{\prime} \\
& \leq\left\|e^{1 / 2} \exp \left(i t a a_{e, f}^{\dagger}\right) e^{-1 / 2}\right\|=\left\|\exp \left(i t a a_{e, f}^{\dagger}\right)\right\|_{e}=1 ; \\
\left\|\exp \left(i t \Pi\left(a_{e, f}^{\dagger}\right) \tilde{a}\right)\right\|_{\tilde{f}}^{\prime} & =\left\|\exp \left(i t \Pi\left(a_{e, f}^{\dagger} a\right)\right)\right\|_{\tilde{f}}^{\prime}=\left\|\Pi\left(f^{1 / 2} \exp \left(i t a_{e, f}^{\dagger} a\right) f^{-1 / 2}\right)\right\|^{\prime} \\
& \leq\left\|f^{1 / 2} \exp \left(i t a_{e, f}^{\dagger} a\right) f^{-1 / 2}\right\|=\left\|\exp \left(i t a_{e, f}^{\dagger} a\right)\right\|_{f}=1 .
\end{aligned}
$$

By [1, Remark 2], the proof is complete.

The weighted Moore-Penrose inverse in closed invariant subspaces will be now studied. To this end, some preparation is needed. Note that if $X$ is a Banach space and $Y \subseteq X$ is a closed invariant subspace for $T \in L(X)$, then $T^{\prime}=\left.T\right|_{Y} \in L(Y)$ stands for the restriction map of $T$ to $Y$.

Proposition 2.12. Let $X$ be a Banach space and consider $U \in L(X)$ an invertible positive operator. Let $Y \subseteq X$ be a closed subspace such that $U(Y)=$ $Y$. Then $U^{\prime} \in L(Y)$ is invertible and positive. What is more, $U^{1 / 2}(Y) \subseteq Y$, $\left(U^{\prime}\right)^{1 / 2}=\left(U^{1 / 2}\right)^{\prime} \in L(Y), U^{-1 / 2}(Y) \subseteq Y$ and $\left(U^{\prime}\right)^{-1 / 2}=\left(U^{-1 / 2}\right)^{\prime} \in L(Y)$.

Proof. Clearly, $U^{-1}(Y)=Y$ and $U^{\prime} \in L(Y)$ is invertible. On the other hand, according to [4, Lemma 5, Chapter I, Section 10], if $S \in A=L(W), W$ a 
Banach space, then $V(S)=\cap_{z \in \mathbb{C}} B[z,\|z-S\|]$, where if $z \in \mathbb{C}$ and $r \in \mathbb{R}_{+}$, then $B[z, r]=\left\{z^{\prime}:\left|z-z^{\prime}\right| \leq r\right\}$. Since

$$
V\left(U^{\prime}\right)=\cap_{z \in \mathbb{C}} B\left[z,\left\|z-U^{\prime}\right\|\right] \subseteq \cap_{z \in \mathbb{C}} B[z,\|z-U\|]=V(U) \subset \mathbb{R}_{+},
$$

$U^{\prime} \in L(Y)$ is positive.

To prove that $\left(U^{\prime}\right)^{1 / 2}=\left(U^{1 / 2}\right)^{\prime} \in L(Y)$, consider $K=\sigma(U) \cup \sigma\left(U^{\prime}\right)$. Since $U \in L(X)$ and $U^{\prime} \in L(Y)$ are invertible and positive, $K \subset G=\mathbb{C} \backslash\{x \in$ $\mathbb{R}: x \leq 0\}$. Let $f: G \rightarrow \mathbb{C}$ be the principal branch of the square root function in $G$, i.e., $f(z)=z^{1 / 2}(z \in G)$. Now, if $\lambda \in G \backslash K$, then according to [7, Lemma 1.28], $(U-\lambda)^{-1}(Y) \subseteq Y$. As a result, according to the formula of the Riesz Functional Calculus for $f: G \rightarrow \mathbb{C}$, using in particular an appropriate system of curves $\Gamma \subseteq G \backslash K$, it is not difficult to prove that $f(U)(Y) \subseteq Y$. However, since $f(U)^{2}=U$ and $\sigma(f(U))=f(\sigma(U)) \subseteq \mathbb{R}_{+} \backslash\{0\}$, according to [8, Theorem], $f(U)=U^{1 / 2}$; in particular, $U^{1 / 2}(Y) \subseteq Y$. Now, since $f\left(U^{\prime}\right)=$ $\left.f(U)\right|_{Y}=\left(U^{1 / 2}\right)^{\prime}, \sigma\left(\left(U^{1 / 2}\right)^{\prime}\right)=\sigma\left(f\left(U^{\prime}\right)\right)=f\left(\sigma\left(U^{\prime}\right)\right) \subseteq R_{+}$. Therefore, since $\left(\left(U^{1 / 2}\right)^{\prime}\right)^{2}=U^{\prime}$, according again to [8, Theorem], $\left(U^{\prime}\right)^{1 / 2}=\left(U^{1 / 2}\right)^{\prime}$.

Concerning the last two facts, note that according to what has been proved and [7, Lemma 1.28], $U^{-1 / 2}(Y) \subseteq Y$. However, a direct calculation, using in particular that $\left(U^{\prime}\right)^{1 / 2}=\left(U^{1 / 2}\right)^{\prime}$, proves that $\left(U^{\prime}\right)^{-1 / 2}=\left(U^{-1 / 2}\right)^{\prime}$.

Theorem 2.13. Let $X$ be a Banach space and consider $E, F, T \in L(X)$ such that $E$ and $F$ are invertible and positive and $T_{E, F}^{\dagger}$ exists. Suppose in addition that there is $Y \subseteq X$ a closed invariant subspace for $T$ and $T_{E, F}^{\dagger}$ such that $E(Y)=Y=F(Y)$. Then the weighted Moore-Penrose inverse of $T^{\prime} \in L(Y)$ with respect to the weights $E^{\prime}, F^{\prime} \in L(Y)$ exists. What is more, $\left(T^{\prime}\right)_{E^{\prime}, F^{\prime}}^{\dagger}=\left(T_{E, F}^{\dagger}\right)^{\prime}$.

Proof. It is clear that $\left(T_{E, F}^{\dagger}\right)^{\prime}$ is a normalized generalized inverse of $T^{\prime}$. On the other hand, according to Proposition 2.12,

$$
\begin{aligned}
\left\|\exp \left(i t T^{\prime}\left(T_{E, F}^{\dagger}\right)^{\prime}\right)\right\|_{E^{\prime}} & =\left\|\left(E^{\prime}\right)^{1 / 2} \exp \left(i t T^{\prime}\left(T_{E, F}^{\dagger}\right)^{\prime}\right)\left(E^{\prime}\right)^{-1 / 2}\right\| \\
& \leq\left\|E^{1 / 2} \exp \left(i t T T_{E, F}^{\dagger}\right) E^{-1 / 2}\right\| \\
& =\left\|\exp \left(i t T T_{E, F}^{\dagger}\right)\right\|_{E}=1, \\
\left\|\exp \left(i t\left(T_{E, F}^{\dagger}\right)^{\prime} T^{\prime}\right)\right\|_{F^{\prime}} & =\left\|\left(F^{\prime}\right)^{1 / 2} \exp \left(i t\left(T_{E, F}^{\dagger}\right)^{\prime} T^{\prime}\right)\left(F^{\prime}\right)^{-1 / 2}\right\| \\
& \leq\left\|F^{1 / 2} \exp \left(i t T_{E, F}^{\dagger} T\right) F^{-1 / 2}\right\| \\
& =\left\|\exp \left(i t T_{E, F}^{\dagger} T\right)\right\|_{F}=1 .
\end{aligned}
$$

Therefore, according to [1, Remark 2], the proof is complete.

The weighted Moore-Penrose inverse in quotient spaces will be studied now. However, some preliminary facts must first be recalled.

Remark 2.14. Let $X$ be a Banach space and consider $U \in L(X)$ an invertible positive operator. Suppose that $Y \subseteq X$ is a closed invariant subspace for $U$ 
and denote by $\Pi: X \rightarrow X / Y$ the quotient map. Then for $\tilde{U} \in L(X / Y)$, the quotient operator induced by $U, \tilde{U}$ is invertible positive. In fact, it is clear that $\tilde{U} \in L(X / Y)$ is invertible. In addition, according to [7, Theorem 4.12(ii)], $\tilde{U}$ is hermitian, and since $\sigma(\tilde{U}) \subseteq \sigma(U) \subset \mathbb{R}_{+}, \tilde{U}$ is positive ([4, Definition 5, Chapter V, Section 38]).

Moreover, according to $\left[8\right.$, Theorem], $(\tilde{U})^{1 / 2}=U^{\tilde{1} / 2}$ and $(\tilde{U})^{-1 / 2}=U^{-1 / 2}$.

Theorem 2.15. Let $X$ be a Banach space and consider $E, F \in L(X)$ two invertible and positive operators. Let $T \in L(X)$ such that $T_{E, F}^{\dagger}$ exists. Suppose in addition that $Y \subseteq X$ is a closed invariant subspace for $T, T_{E, F}^{\dagger}, E$ and $F$. Then $\tilde{T} \in L(X / Y)$ is weighted Moore-Penrose invertible with weights $\tilde{E}$ and $\tilde{F}$. Furthermore, $(\tilde{T})_{\tilde{E}, \tilde{F}}^{\dagger}=T_{E, F}^{\tilde{\dagger}}$.

Proof. It is clear that $T_{E, F}^{\tilde{\dagger}}$ is a normalized generalized inverse of $\tilde{T}$. On the other hand,

$$
\begin{aligned}
\left\|\exp \left(i t \tilde{T} T_{E, F}^{\tilde{\dagger}}\right)\right\|_{\tilde{E}} & =\left\|\tilde{E}^{1 / 2} \exp \left(i t \tilde{T} T_{E, F}^{\tilde{\dagger}}\right) \tilde{E}^{-1 / 2}\right\| \\
& =\left\|\Pi\left(E^{1 / 2} \exp \left(i t T T_{E, F}^{\dagger}\right) E^{-1 / 2}\right)\right\| \\
& \leq\left\|E^{1 / 2} \exp \left(i t T T_{E, F}^{\dagger}\right) E^{-1 / 2}\right\|=\left\|\exp \left(i t T T_{E, F}^{\dagger}\right)\right\|_{E}=1 \\
\left\|\exp \left(i t T_{E, F}^{\tilde{\dagger}} \tilde{T}\right)\right\|_{\tilde{F}} & =\left\|\tilde{F}^{1 / 2} \exp \left(i t T_{E, F}^{\tilde{\dagger}} \tilde{T}\right) \tilde{F}^{-1 / 2}\right\| \\
& =\left\|\Pi\left(F^{1 / 2} \exp \left(i t T_{E, F}^{\dagger} T\right) F^{-1 / 2}\right)\right\| \\
& \leq\left\|F^{1 / 2} \exp \left(i t T_{E, F}^{\dagger} T\right) F^{-1 / 2}\right\|=\left\|\exp \left(i t T_{E, F}^{\dagger} T\right)\right\|_{F}=1
\end{aligned}
$$

Consequently, according to $[1, \operatorname{Remark} 2],(\tilde{T})_{\tilde{E}, \tilde{F}}^{\dagger}=T_{E, F}^{\tilde{\dagger}}$.

\section{Weighted EP elements}

In this section, weighted EP Banach space operators and Banach algebra elements will be considered. In particular, these elements will be characterized extending the results for matrices ([24]) and elements of $C^{*}$-algebras ([18]). First, the main notion of this section will be introduced.

Definition 3.1. Given a unital Banach algebra $A$ and $e, f \in A$ two invertible positive elements, $a \in A$ is said to be weighted EP with weights $e$ and $f$ if $a_{e, f}^{\dagger}$ exists and commutes with $a$.

Under the same conditions as in Definition 3.1, as it was pointed out in the paragraph that follows Definition 2.3, note that if $e=f$, then the necessary and sufficient condition for $a \in A$ to be weighted $\mathrm{EP}$ with weight $e$ is that $a \in A^{e}$ is EP; EP Banach algebra elements are studied in $[1,2,3,17]$. In the context of $C^{*}$-algebras, see [13]. 
To study the objects introduced in Definition 3.1, the notion of group inverse need to be recalled. In fact, weighted EP Banach algebra elements consists in a particular class of group invertible elements.

Definition 3.2. Given a unital Banach algebra $A$ and $a \in A$, an element $b \in A$ is said to be the group inverse of $a$ if the following set of equations is satisfied:

$$
a=a b a, b=b a b, a b=b a .
$$

Under the conditions of Definition 3.2, note that according to $[9$, Theorem $9]$, if the group inverse of $a \in A$ exists, then it is unique. In this case, the group inverse of $a \in A$ is denoted by $a^{\sharp}$. In the following remark, some of the most relevant properties of group inverses will be recalled.

Remark 3.3. (i) Let $A$ be a unital Banach algebra and consider $a \in A$. Suppose that $b \in A$ is a normalized generalized inverse of $a$. Then the necessary and sufficient condition for $b$ to be the group inverse of $a$ is that $L_{b} \in L(A)$ (respectively, $R_{b} \in L(A)$ ) is the group inverse of $L_{a} \in L(A)$ (respectively, $R_{a} \in L(A)$ ). In fact, since $L_{b}$ is a normalized generalized inverse of $L_{a}$, according to Definition 3.2, the statement under consideration is equivalent to proving that $a$ and $b$ commute if and only if $L_{a}$ and $L_{b}$ commute, which is clear. A similar argument proves the statement for the right multiplication operator on $L(A)$. In addition, note that in this case, according to [9, Theorem 9], $\left(L_{a}\right)^{\sharp}=L_{a^{\sharp}}$ (respectively, $\left(R_{a}\right)^{\sharp}=R_{a^{\sharp}}$ ).

(ii) Suppose that $e, f \in A$ are invertible and positive ( $A$ as in (i)). Note that if $a \in A$ is group invertible, then the necessary and sufficient condition for $a$ to be weighted EP with weights $e$ and $f$ is that $a a^{\sharp} \in A^{e}$ and $a^{\sharp} a \in A^{f}$ are hermitian. In fact, if $a \in A$ is weighted EP with weights $e$ and $f$, then according to $\left[9\right.$, Theorem 9], $a^{\sharp}$ exists, which is actually $a^{\sharp}=a_{e, f}^{\dagger}$. In particular, $a a^{\sharp} \in A^{e}$ and $a^{\sharp} a \in A^{f}$ are hermitian. On the other hand, if $a a^{\sharp} \in A^{e}$ and $a^{\sharp} a \in A^{f}$ are hermitian, then according to Definition 2.3, $a_{e, f}^{\dagger}$ exists. What is more, according to Proposition 2.5, $a^{\sharp}=a_{e, f}^{\dagger}$. Since $a a_{e, f}^{\dagger}=a a^{\sharp}=a^{\sharp} a=a_{e, f}^{\dagger} a, a$ is weighted EP with weights $e$ and $f$. Consequently, weighted EP elements are group invertible elements for which the weighted Moore-Penrose inverse exists and coincides with the group inverse.

(iii) Let $A=L(X), X$ a Banach space, and consider $T \in L(X)$. Then according to $[11$, Lemma 1$]$, the necessary and sufficient condition for $T^{\sharp}$ to exist is that $X=N(T) \oplus R(T)$.

(iv) Under the conditions of (iii), note that if $T \in L(X)$ is group invertible, then, as in Remark 2.6(b), it is not difficult to prove that $N(T)=N\left(T^{\sharp} T\right)=$ $N\left(T T^{\sharp}\right)=N\left(T^{\sharp}\right)$ and $R(T)=R\left(T T^{\sharp}\right)=R\left(T^{\sharp} T\right)=R\left(T^{\sharp}\right)$.

(v) Observe that according to items (iii) and (iv), $R\left(T^{k}\right)=R\left(\left(T^{\sharp}\right)^{k}\right)=R(T)$ and $N\left(T^{k}\right)=N\left(\left(T^{\sharp}\right)^{k}\right)=N(T)$.

In the following theorem, the first characterization of weighted EP elements will be given. 
Theorem 3.4. (a) Let $X$ be a Banach space and consider $E, F \in L(X)$ two invertible positive operators. Then for $T \in L(X)$, the following statements are equivalent.

(i) $T$ is weighted $E P$ with weights $E$ and $F$;

(ii) there exists an idempotent $P \in L(X)$ such that $P \in H\left(L(X)^{E}\right) \cap$ $H\left(L(X)^{F}\right), R(P)=R(T)$ and $N(P)=N(T)$.

(b) Let $A$ be a unital Banach algebra and consider $e, f \in A$ two invertible positive elements. Then for $a \in A$ such that $a_{e, f}^{\dagger}$ exists, the following statements are equivalent.

(iii) $a$ is weighted EP with weights $e$ and $f$;

(iv) there exists an idempotent $P \in L(A)$ such that $P \in H\left(L(A)^{L_{e}}\right) \cap$ $H\left(L(A)^{L_{f}}\right), R(P)=a A$ and $N(P)=a^{-1}(0)$;

(v) $L_{a} \in L(A)$ is weighted $E P$ with weights $L_{e}$ and $L_{f}$.

Furthermore, if in (a) or (b) such $P$ exists, then it is unique.

Proof. (a) If $T$ is weighted EP with weights $E$ and $F$, then $P=T T_{E, F}^{\dagger}=T_{E, F}^{\dagger} T$ satisfies the required property (Remark 2.6(b)).

On the other hand, if statement (ii) holds, then according to Theorem 2.7, $T_{E, F}^{\dagger}$ exists. Further, observe that $R\left(T T_{E, F}^{\dagger}\right)=R(T)=R(P)$ and $R(I-$ $\left.T_{E, F}^{\dagger} T\right)=N\left(T_{E, F}^{\dagger} T\right)=N(T)=N(P)=R(I-P)$. Therefore, according to [25, Hilfssatz 2(a)-(b)] and [20, Theorem 2.2], $T_{E, F}^{\dagger} T=P=T T_{E, F}^{\dagger}$.

(b) According to what has been proved, statements (iv) and (v) are equivalent. In addition, since according to Theorem 2.8, $\left(L_{a}\right)_{L_{e}, L_{f}}^{\dagger}=L_{a_{e, f}^{\dagger}}$, statements (iii) and (v) are equivalent.

The last statement is a consequence of Theorem 2.7.

Next, some basic characterizations of weighted EP Banach space operators will be given.

Theorem 3.5. Let $X$ be a Banach space and consider $E, F \in L(X)$ two invertible positive operators. Suppose, in addition, that $T \in L(X)$ such that $T_{E, F}^{\dagger}$ and $T^{\sharp}$ exist. Then the following statements are equivalent.

(i) $T$ is weighted $E P$ with weights $E$ and $F$;

(ii) $R\left(T_{E, F}^{\dagger}\right)=R(T)$ and $N\left(T_{E, F}^{\dagger}\right)=N(T)$;

(iii) $R\left(T_{E, F}^{\dagger}\right) \subset R(T)$ and $N(T) \subset N\left(T_{E, F}^{\dagger}\right)$;

(iv) $R(T) \subset R\left(T_{E, F}^{\dagger}\right)$ and $N(T) \subset N\left(T_{E, F}^{\dagger}\right)$;

(v) $R\left(T_{E, F}^{\dagger}\right) \subset R(T)$ and $N\left(T_{E, F}^{\dagger}\right) \subset N(T)$;

(vi) $R(T) \subset R\left(T_{E, F}^{\dagger}\right)$ and $N\left(T_{E, F}^{\dagger}\right) \subset N(T)$;

(vii) $T_{E, F}^{\dagger}=T^{\sharp}$;

(viii) $T_{E, F}^{\dagger}=T\left(T_{E, F}^{\dagger}\right)^{2}=\left(T_{E, F}^{\dagger}\right)^{2} T$;

(ix) $T=T_{E, F}^{\dagger} T^{2}=T^{2} T_{E, F}^{\dagger}$;

(x) $T_{E, F}^{\dagger}$ is weighted EP with weights $F$ and $E$; moreover, $\left(T_{E, F}^{\dagger}\right)_{F, E}^{\dagger}=T$; 
(xi) $T$ is weighted $E P$ with weights $F$ and $E$;

(xii) $T$ is both weighted $E P$ with weights $E$ and $E$ and with weights $F$ and $F$;

(xiii) $T^{k}$ is weighted $E P$ with weights $E$ and $F$ for some integer $k \geq 1$;

(xiv) $T^{\sharp}$ is weighted $E P$ with weights $E$ and $F$;

(xv) $T T^{\sharp}=T T_{E, E}^{\dagger}=T T_{F, F}^{\dagger}\left(\right.$ or $\left.T T^{\sharp}=T_{E, E}^{\dagger} T=T_{F, F}^{\dagger} T\right)$;

(xvi) $T T^{\sharp}=T T_{E, F}^{\dagger}=T T_{F, E}^{\dagger}\left(\right.$ or $\left.T T^{\sharp}=T_{F, E}^{\dagger} T=T_{E, F}^{\dagger} T\right)$.

Proof. If statement (i) holds, then according to Remark 2.6(b), $R\left(T_{E, F}^{\dagger}\right)=$ $R\left(T_{E, F}^{\dagger} T\right)=R\left(T T_{E, F}^{\dagger}\right)=R(T)$ and $N\left(T_{E, F}^{\dagger}\right)=N\left(T T_{E, F}^{\dagger}\right)=N\left(T_{E, F}^{\dagger} T\right)=$ $N(T)$. Consequently, statement (ii) holds, which in turn clearly implies statements (iii)-(vi).

On the other hand, if one of the statements (iii)-(vi) holds, say statement (iii) for example, then using the decompositions of $X$

$$
X=R\left(T_{E, F}^{\dagger}\right) \oplus N(T)=R(T) \oplus N\left(T_{E, F}^{\dagger}\right)=R(T) \oplus N(T)
$$

(Remark 2.6(b) and Remark 3.3(iii)), it is not difficult to prove that $R\left(T_{E, F}^{\dagger}\right)=$ $R(T)$ and $N\left(T_{E, F}^{\dagger}\right)=N(T)$, or equivalently, statement (ii) holds. However, in this case, $R\left(T_{E, F}^{\dagger} T\right)=R\left(T T_{E, F}^{\dagger}\right)$ and $N\left(T_{E, F}^{\dagger} T\right)=N\left(T T_{E, F}^{\dagger}\right)$. Since $T_{E, F}^{\dagger} T$ and $T T_{E, F}^{\dagger} \in L(X)$ are idempotents, $T_{E, F}^{\dagger} T=T T_{E, F}^{\dagger}$, i.e., $T$ is weighted EP with weights $E$ and $F$.

An argument similar to that in Remark 3.3(ii) proves the equivalence between statements (i) and (vii).

Statement (i) implies statements (viii)-(ix). On the other hand, statement (viii) implies statement (iii) and statement (ix) implies statement (vi).

The equivalence between statements (i) and ( $\mathrm{x}$ ) is a consequence of Definition 2.3 .

Since according to Remark 3.3(ii), $T$ is weighted EP with weights $F$ and $E$ if and only if $T T^{\sharp} \in H\left(L(X)^{F}\right) \cap H\left(L(X)^{E}\right)$, statements (i) and (xi) are equivalent. Similarly, it is possible to prove that statement (i) is equivalent to statements (xii)-(xiv) using that $T^{k}\left(T^{k}\right)^{\sharp}=T^{k}\left(T^{\sharp}\right)^{k}=T T^{\sharp}$ (for some integer $k \geq 1)$ and $\left(T^{\sharp}\right)^{\sharp}=T^{\sharp}$.

If statement (xii) holds, then $T^{\sharp}=T_{E, E}^{\dagger}=T_{F, F}^{\dagger}$ and statement (xv) is satisfied, which in turn implies statement (i) (Remark 3.3(ii)). In a similar way, it is possible to prove that statement (xvi) implies statement (i) and statement (xi) implies statement (xvi).

In the following theorem, more characterizations concerning weighted EP operators will be proved.

Theorem 3.6. Let $X$ be a Banach space and consider $E, F \in L(X)$ two invertible positive operators. Suppose, in addition, that $T \in L(X)$ such that $T_{E, F}^{\dagger}$ and $T^{\sharp}$ exist. Then the necessary and sufficient condition for $T$ to be weighted 
$E P$ with weights $E$ and $F$ is that one of the following statements holds.

(i) $\quad T T^{\sharp} T_{E, F}^{\dagger}=T_{E, F}^{\dagger} T^{\sharp} T$;

(ii) $\quad T T_{E, F}^{\dagger} T_{E, F}^{\dagger} T=T_{E, F}^{\dagger} T T T_{E, F}^{\dagger}$;

(iii) $\quad T_{E, F}^{\dagger} T^{\sharp} T+T T^{\sharp} T_{E, F}^{\dagger}=2 T_{E, F}^{\dagger}$;

(iv) $\quad\left(T_{E, F}^{\dagger}\right)^{2} T^{\sharp}=T_{E, F}^{\dagger} T^{\sharp} T_{E, F}^{\dagger}=T^{\sharp}\left(T_{E, F}^{\dagger}\right)^{2}$;

(v) $\quad T\left(T_{E, F}^{\dagger}\right)^{2}=T^{\sharp}=\left(T_{E, F}^{\dagger}\right)^{2} T$;

(vi) $\quad T^{k}=T_{E, F}^{\dagger} T T^{k}=T^{k} T T_{E, F}^{\dagger}$;

(vii) $\quad\left(T_{E, F}^{\dagger}\right)^{k}=\left(T^{\sharp}\right)^{k}$;

(viii) $\quad\left(T^{\sharp}\right)^{k} T_{E, F}^{\dagger}=T_{E, F}^{\dagger}\left(T^{\sharp}\right)^{k}$;

(ix) $\quad T^{k} T_{E, F}^{\dagger}=T_{E, F}^{\dagger} T^{k}$;

(x) $\quad T^{2 k-1}=T_{E, F}^{\dagger} T^{2 k+1} T_{E, F}^{\dagger}$;

(xi) $\quad\left(T^{\sharp}\right)^{k} T_{E, F}^{\dagger} T=\left(T_{E, F}^{\dagger}\right)^{k}$;

(xii) $\quad T\left(T_{E, F}^{\dagger}\right)^{k+1}=\left(T^{\sharp}\right)^{k}=\left(T_{E, F}^{\dagger}\right)^{k+1} T$;

(xiii) $\quad T^{k} T T_{E, F}^{\dagger}+T_{E, F}^{\dagger} T T^{k}=2 T^{k}$;

(xiv) $\quad\left(T^{\sharp}\right)^{k+l-1}=\left(T_{E, F}^{\dagger}\right)^{l}\left(T^{\sharp}\right)^{k-1}=\left(T^{\sharp}\right)^{k-1}\left(T_{E, F}^{\dagger}\right)^{l}$;

(xv) $\quad T T_{E, F}^{\dagger}\left(T^{\sharp}\right)^{k+l-1}=\left(T_{E, F}^{\dagger}\right)^{k}\left(T^{\sharp}\right)^{l} T=\left(T^{\sharp}\right)^{l} T\left(T_{E, F}^{\dagger}\right)^{k}$;

(xvi) $\quad T T_{E, F}^{\dagger}\left(T^{k}+\lambda T_{E, F}^{\dagger}\right)=\left(T^{k}+\lambda T_{E, F}^{\dagger}\right) T T_{E, F}^{\dagger} ;$

(xvii) $\quad T_{E, F}^{\dagger} T\left(T^{k}+\lambda T_{E, F}^{\dagger}\right)=\left(T^{k}+\lambda T_{E, F}^{\dagger}\right) T_{E, F}^{\dagger} T$;

(xviii) $\quad R\left(T+\lambda T_{E, E}^{\dagger}\right)=R\left(T+\lambda T_{F, F}^{\dagger}\right)=R\left(\lambda T+T^{3}\right)$ and

$N\left(T+\lambda T_{E, E}^{\dagger}\right)=N\left(T+\lambda T_{F, F}^{\dagger}\right)=N\left(\lambda T+T^{3}\right) ;$

(xix) $\quad R\left(T+\lambda T_{E, F}^{\dagger}\right)=R\left(\lambda T+T^{3}\right)$ and $N\left(T+\lambda T_{E, F}^{\dagger}\right)=N\left(\lambda T+T^{3}\right)$,

where $k, l \in \mathbb{N}$ and $\lambda \in \mathbb{C} \backslash\{0\}$.

Proof. It is not difficult to prove that the condition of being weighted EP with weights $E$ and $F$ implies statements (i)-(xvii). On the other hand, if statement (i) holds, then

$$
R\left(T T^{\sharp} T_{E, F}^{\dagger}\right)=R\left(T^{\sharp} T T_{E, F}^{\dagger}\right)=T^{\sharp} R\left(T T_{E, F}^{\dagger}\right)=R(T) .
$$

As a result, $R(T) \subseteq R\left(T_{E, F}^{\dagger}\right)$. In addition, if $x \in N\left(T_{E, F}^{\dagger}\right)$, then $T^{\sharp} T(x) \in$ $R(T) \cap N\left(T_{E, F}^{\dagger}\right)=\{0\}$, Thus $x \in N\left(T^{\sharp} T\right)=N(T)$ and $N\left(T_{E, F}^{\dagger}\right) \subseteq N(T)$. Consequently, according to Theorem 3.5(vi), the condition of being weighted $\mathrm{EP}$ is satisfied. 
Suppose that statement (ii) holds. If $x \in N(T)$, then $T T_{E, F}^{\dagger}(x) \in R(T) \cap$ $N\left(T_{E, F}^{\dagger} T\right)=R(T) \cap N(T)=\{0\}$. Thus $x \in N\left(T T_{E, F}^{\dagger}\right)=N\left(T_{E, F}^{\dagger}\right)$ and $N(T) \subseteq N\left(T_{E, F}^{\dagger}\right)$. On the other hand, since

$R\left(T_{E, F}^{\dagger} T T T_{E, F}^{\dagger}\right)=T_{E, F}^{\dagger} T\left(R\left(T T_{E, F}^{\dagger}\right)\right)=T_{E, F}^{\dagger} T(R(T))=R\left(T_{E, F}^{\dagger} T\right)=R\left(T_{E, F}^{\dagger}\right)$, $R\left(T_{E, F}^{\dagger}\right) \subseteq R(T)$. Therefore, according to Theorem 3.5(iii), $T$ is weighted EP with weights $E$ and $F$.

If statement (iii) holds, then $R(T)=R\left(T T_{E, F}^{\dagger}\right)=R\left(T^{\sharp} T T_{E, F}^{\dagger}\right) \subseteq R\left(T_{E, F}^{\dagger}\right)$. In addition, if $x \in N\left(T_{E, F}^{\dagger}\right)$, then $T^{\sharp} T(x) \in R(T) \cap N\left(T_{E, F}^{\dagger}\right)=\{0\}$. Thus $x \in N\left(T^{\sharp} T\right)=N(T)$ and $N\left(T_{E, F}^{\dagger}\right) \subseteq N(T)$. In particular, according to Theorem 3.5(vi), the condition of being weighted EP is satisfied.

Suppose that statement (iv) holds. According to the equality $\left(T_{E, F}^{\dagger}\right)^{2} T^{\sharp}=$ $T_{E, F}^{\dagger} T^{\sharp} T_{E, F}^{\dagger}$,

$$
\begin{aligned}
\left(T_{E, F}^{\dagger}\right)^{2} T^{\sharp} & =\left(\left(T_{E, F}^{\dagger}\right)^{2} T^{\sharp}\right) T T^{\sharp}=T_{E, F}^{\dagger} T^{\sharp} T_{E, F}^{\dagger} T T^{\sharp} \\
& =T_{E, F}^{\dagger}\left(T^{\sharp}\right)^{2} T T_{E, F}^{\dagger} T T^{\sharp}=T_{E, F}^{\dagger}\left(T^{\sharp}\right)^{2} .
\end{aligned}
$$

As a result,

$$
\begin{aligned}
T T_{E, F}^{\dagger} & =T^{3}\left(T^{\sharp}\right)^{2} T_{E, F}^{\dagger}=T^{3} T_{E, F}^{\dagger} T\left(T^{\sharp}\right)^{2} T_{E, F}^{\dagger}=T^{3}\left(T_{E, F}^{\dagger} T^{\sharp} T_{E, F}^{\dagger}\right) \\
& =T^{3}\left(\left(T_{E, F}^{\dagger}\right)^{2} T^{\sharp}\right)=T^{3} T_{E, F}^{\dagger}\left(T^{\sharp}\right)^{2}=T^{3} T_{E, F}^{\dagger} T\left(T^{\sharp}\right)^{3}=T T^{\sharp} .
\end{aligned}
$$

A similar argument, using in particular the equality

$$
T_{E, F}^{\dagger} T^{\sharp} T_{E, F}^{\dagger}=T^{\sharp}\left(T_{E, F}^{\dagger}\right)^{2},
$$

proves that $T_{E, F}^{\dagger} T=T T^{\sharp}$. Therefore, $T$ is weighted $\mathrm{EP}$ with weights $E$ and $F$.

Next consider statement (v). The condition $T\left(T_{E, F}^{\dagger}\right)^{2}=T^{\sharp}=\left(T_{E, F}^{\dagger}\right)^{2} T$ implies that

$$
T T_{E, F}^{\dagger}=T T^{\sharp} T T_{E, F}^{\dagger}=T T\left(T_{E, F}^{\dagger}\right)^{2} T T_{E, F}^{\dagger}=T\left(T\left(T_{E, F}^{\dagger}\right)^{2}\right)=T T^{\sharp}
$$

and

$$
T_{E, F}^{\dagger} T=T_{E, F}^{\dagger} T T^{\sharp} T=T_{E, F}^{\dagger} T\left(T_{E, F}^{\dagger}\right)^{2} T T=\left(\left(T_{E, F}^{\dagger}\right)^{2} T\right) T=T^{\sharp} T .
$$

Consequently, $T$ is weighted EP with weights $E$ and $F$.

If statement (vi) holds, then $R(T)=R\left(T^{k}\right) \subseteq R\left(T_{E, F}^{\dagger}\right)$ and $N\left(T_{E, F}^{\dagger}\right) \subseteq$ $N\left(T^{k}\right)=N(T)$. Therefore, according to Theorem 3.5(vi), $T$ is weighted EP with weights $E$ and $F$. A similar argument, using in particular that the ranges and null spaces of $T$ and $T^{\sharp}$ coincide, proves that statement (vii) is equivalent to the condition of being weighted EP.

Suppose that statement (viii) holds. Then

$$
R\left(T_{E, F}^{\dagger}\right)=R\left(T_{E, F}^{\dagger} T\right)=T_{E, F}^{\dagger}(R(T))
$$




$$
\begin{aligned}
& =T_{E, F}^{\dagger}\left(R\left(\left(T^{\sharp}\right)^{k}\right)\right)=R\left(T_{E, F}^{\dagger}\left(T^{\sharp}\right)^{k}\right) \\
& \subseteq R\left(\left(T^{\sharp}\right)^{k}\right)=R(T) .
\end{aligned}
$$

In addition, if $x \in N(T)=N\left(\left(T^{\sharp}\right)^{k}\right)$, then $T_{E, F}^{\dagger}(x) \in R\left(T_{E, F}^{\dagger}\right) \cap N\left(\left(T^{\sharp}\right)^{k}\right)=$ $R\left(T_{E, F}^{\dagger}\right) \cap N(T)=\{0\}$. As a result, $N(T) \subseteq N\left(T_{E, F}^{\dagger}\right)$. Consequently, according to Theorem 3.5(iii), $T$ is weighted EP with weights $E$ and $F$. A similar argument can be applied to statement (ix).

Statement (x) implies that $N\left(T_{E, F}^{\dagger}\right) \subseteq N\left(T^{2 k-1}\right)=N(T)$ and $R(T)=$ $R\left(T^{2 k-1}\right) \subseteq R\left(T_{E, F}^{\dagger}\right)$. As a result, according to Theorem 3.5(vi), the condition of being weighted EP is satisfied.

Suppose that statement (xi) holds. Now, since $X=R\left(T_{E, F}^{\dagger} T\right) \oplus N\left(\left(T^{\sharp}\right)^{k}\right)$, $R(T)=R\left(\left(T^{\sharp}\right)^{k}\right) \subseteq R\left(T_{E, F}^{\dagger}\right)$. In addition, if $x \in N\left(T_{E, F}^{\dagger}\right)$, then $T_{E, F}^{\dagger} T(x) \in$ $R\left(T_{E, F}^{\dagger}\right) \cap N\left(\left(T^{\sharp}\right)^{k}\right)=R\left(T_{E, F}^{\dagger}\right) \cap N(T)=\{0\}$. Hence $x \in N\left(T_{E, F}^{\dagger} T\right)=$ $N(T)$ and $N\left(T_{E, F}^{\dagger}\right) \subseteq N(T)$. Consequently, according to Theorem 3.5(vi), $T$ is weighted $\mathrm{EP}$ with weights $E$ and $F$.

Suppose that statement (xii) holds. Then it is not difficult to prove that $R(T) \subseteq R\left(T_{E, F}^{\dagger}\right)$ and $N\left(T_{E, F}^{\dagger}\right) \subseteq N(T)$. In particular, according to Theorem 3.5 (vi), $T$ is weighted EP with weights $E$ and $F$.

If statement (xiii) holds, then $R\left(T_{E, F}^{\dagger}\right)=R\left(T_{E, F}^{\dagger} T\right)=R\left(T_{E, F}^{\dagger} T T^{k}\right) \subseteq$ $R\left(T^{k}\right)=R(T)$. In addition, if $x \in N(T)=N\left(T^{k}\right)$, then $T_{E, F}^{\dagger}(x) \in N\left(T^{k+1}\right)=$ $N(T)$, which implies that $x \in N\left(T_{E, F}^{\dagger}\right)$. Thus $N(T) \subseteq N\left(T_{E, F}^{\dagger}\right)$. However, according to Theorem 3.5(iii), the condition of being weighted EP holds.

Statement (xiv) implies that $R(T) \subseteq R\left(T_{E, F}^{\dagger}\right.$ ) and $\left.N\left(T_{E, F}^{\dagger}\right)\right) \subseteq N(T)$. Therefore, according to Theorem 3.5(vi), $T$ is weighted EP with weights $E$ and $F$.

Suppose that statement (xv) holds. Then

$$
\begin{aligned}
R(T) & =R\left(T T_{E, F}^{\dagger} T\right)=T T_{E, F}^{\dagger} R(T) \\
& =T T_{E, F}^{\dagger} R\left(\left(T^{\sharp}\right)^{k+l-1}\right)=R\left(T T_{E, F}^{\dagger}\left(T^{\sharp}\right)^{k+l-1}\right) \\
& \subseteq R\left(T_{E, F}^{\dagger}\right) .
\end{aligned}
$$

In addition, if $x \in N\left(T_{E, F}^{\dagger}\right)$, then

$$
\left(T^{\sharp}\right)^{k+l-1}(x) \in N\left(T T_{E, F}^{\dagger}\right) \cap R(T)=N\left(T_{E, F}^{\dagger}\right) \cap R(T)=\{0\} .
$$

Then $N\left(T_{E, F}^{\dagger}\right) \subseteq N(T)$. Therefore, according to Theorem 3.5(vi), the condition of being weighted EP is satisfied.

Statement (xvi) can be rewritten as

$$
T^{k}+\lambda T T_{E, F}^{\dagger} T_{E, F}^{\dagger}=T^{k} T T_{E, F}^{\dagger}+\lambda T_{E, F}^{\dagger} .
$$


Then, multiplying (1) from the left side by $T T_{E, F}^{\dagger}$,

$$
T^{k}+\lambda T T_{E, F}^{\dagger} T_{E, F}^{\dagger}=T^{k} T T_{E, F}^{\dagger}+\lambda T T_{E, F}^{\dagger} T_{E, F}^{\dagger} .
$$

Hence $T^{k}=T^{k} T T_{E, F}^{\dagger}$, which implies that $N\left(T_{E, F}^{\dagger}\right) \subseteq N\left(T^{k}\right)=N(T)$. Similarly, multiplying (1) from the right side by $T T_{E, F}^{\dagger}$,

$$
T^{k} T T_{E, F}^{\dagger}+\lambda T T_{E, F}^{\dagger} T_{E, F}^{\dagger}=T^{k} T T_{E, F}^{\dagger}+\lambda T_{E, F}^{\dagger} .
$$

As a result, $T T_{E, F}^{\dagger} T_{E, F}^{\dagger}=T_{E, F}^{\dagger}$, which implies that $R\left(T_{E, F}^{\dagger}\right) \subseteq R(T)$. Consequently, according to Theorem $3.5(\mathrm{v}), T$ is weighted EP with weights $E$ and $F$.

A similar argument proves that statement (xvii) implies statement (i).

Suppose that statements (xviii) holds. The equality $R\left(T+\lambda T_{E, E}^{\dagger}\right)=R(\lambda T+$ $\left.T^{3}\right)$ implies that for $x \in X$, there exists $y \in X$ such that $\left(T+\lambda T_{E, E}^{\dagger}\right) x=$ $\left(\lambda T+T^{3}\right) y$. Then

$$
\begin{aligned}
\left(T+\lambda T T_{E, E}^{\dagger} T_{E, E}^{\dagger}\right) x & =T T_{E, E}^{\dagger}\left(T+\lambda T_{E, E}^{\dagger}\right) x=T T_{E, E}^{\dagger}\left(\lambda T+T^{3}\right) y \\
& =\left(\lambda T+T^{3}\right) y=\left(T+\lambda T_{E, E}^{\dagger}\right) x,
\end{aligned}
$$

which implies that $T T_{E, E}^{\dagger} T_{E, E}^{\dagger} x=T_{E, E}^{\dagger} x(x \in X)$. Thus $R\left(T_{E, E}^{\dagger}\right) \subseteq R(T)$. Let $x \in N(T)$. Now, $\left(\lambda T+T^{3}\right) x=0$ and since $N\left(T+\lambda T_{E, E}^{\dagger}\right)=N\left(\lambda T+T^{3}\right)$, $\left(T+\lambda T_{E, E}^{\dagger}\right) x=0$, i.e., $T_{E, E}^{\dagger} x=0$. Therefore, $N(T) \subseteq N\left(T_{E, E}^{\dagger}\right)$, and according to Theorem 3.5(iii), $T$ is weighted EP with weights $E$ and $E$. Similarly, using that $R\left(T+\lambda T_{F, F}^{\dagger}\right)=R\left(\lambda T+T^{3}\right)$ and $N\left(T+\lambda T_{F, F}^{\dagger}\right)=N\left(\lambda T+T^{3}\right)$, it is possible to prove that that $T$ is weighted EP with weights $F$ and $F$. According then to Theorem 3.5(xii), $T$ is weighted EP with weights $E$ and $F$.

On the other hand, if $T$ is weighted EP with weights $E$ and $F$, then $T^{\sharp}=$ $T_{E, E}^{\dagger}=T_{F, F}^{\dagger}$ (Theorem 3.5(xii)). Since

$$
T+\lambda T^{\sharp}=\left(T^{3}+\lambda T\right)\left(T^{\sharp}\right)^{2}=\left(T^{\sharp}\right)^{2}\left(T^{3}+\lambda T\right)
$$

and

$$
T^{3}+\lambda T=\left(T+\lambda T^{\sharp}\right) T^{2}=T^{2}\left(T+\lambda T^{\sharp}\right),
$$

statement (xviii) holds. A similar argument proves that statement (xix) is equivalent to the condition of being weighted EP.

Next, weighted EP Banach algebra elements will be characterized.

Theorem 3.7. Let $A$ be a unital Banach algebra and consider $e, f \in A$ two invertible positive elements. Suppose, in addition, that $a \in A$ such that $a_{e, f}^{\dagger}$ and $a^{\sharp}$ exist. Then the following statements are equivalent.

(i) $a$ is weighted $E P$ with weights $e$ and $f$;

(ii) $a_{e, f}^{\dagger} A=a A$ and $\left(a_{e, f}^{\dagger}\right)^{-1}(0)=a^{-1}(0)$;

(iii) $a_{e, f}^{\dagger} A \subset a A$ and $a^{-1}(0) \subset\left(a_{e, f}^{\dagger}\right)^{-1}(0)$; 
(iv) $a A \subset a_{e, f}^{\dagger} A$ and $a^{-1}(0) \subset\left(a_{e, f}^{\dagger}\right)^{-1}(0)$;

(v) $a_{e, f}^{\dagger} A \subseteq a A$ and $\left(a_{e, f}^{\dagger}\right)^{-1}(0) \subset a^{-1}(0)$;

(vi) $a A \subset a_{e, f}^{\dagger} A$ and $\left(a_{e, f}^{\dagger}\right)^{-1}(0) \subset a^{-1}(0)$;

(vii) $a_{e, f}^{\dagger}=a\left(a_{e, f}^{\dagger}\right)^{2}=\left(a_{e, f}^{\dagger}\right)^{2} a$;

(viii) $a_{e, f}^{\dagger}$ is weighted EP with weights $f$ and $e$;

(ix) $a_{e, f}^{\dagger} a^{\sharp} a+a a^{\sharp} a_{e, f}^{\dagger}=2 a_{e, f}^{\dagger}$;

(x) $\left(a_{e, f}^{\dagger}\right)^{2} a^{\sharp}=a_{e, f}^{\dagger} a^{\sharp} a_{e, f}^{\dagger}=a^{\sharp}\left(a_{e, f}^{\dagger}\right)^{2}$;

(xi) $a\left(a_{e, f}^{\dagger}\right)^{2}=a^{\sharp}=\left(a_{e, f}^{\dagger}\right)^{2} a$;

(xii) $a a_{e, f}^{\dagger} a_{e, f}^{\dagger} a=a_{e, f}^{\dagger} a a a_{e, f}^{\dagger}$;

(xiii) $a a^{\sharp} a_{e, f}^{\dagger}=a_{e, f}^{\dagger} a^{\sharp} a$;

(xiv) $a \in a_{e, f}^{\dagger} A^{-1} \cap A^{-1} a_{e, f}^{\dagger}$;

(xv) there exist $u, v \in A$ such that $a=a_{e, f}^{\dagger} u=v a_{e, f}^{\dagger}, u A=A$ and $v^{-1}(0)=$ $\{0\}$;

(xvi) $a \in a_{e, f}^{\dagger} A \cap A a_{e, f}^{\dagger}$;

(xvii) if $b \in A$ such that $a b=b a$, then $a_{e, f}^{\dagger} b=b a_{e, f}^{\dagger}$;

(xviii) there exists a holomorphic function $f: U \rightarrow \mathbb{C}, \sigma(a) \subseteq U$, such that $a_{e, f}^{\dagger}=f(a)$

(xix) $\left(a^{\sharp}\right)^{k} a_{e, f}^{\dagger} a=\left(a_{e, f}^{\dagger}\right)^{k}$;

$(\mathrm{xx}) a^{k}=a_{e, f}^{\dagger} a a^{k}=a^{k} a a_{e, f}^{\dagger}$;

(xxi) $\left(a_{e, f}^{\dagger}\right)^{k}=\left(a^{\sharp}\right)^{k}$;

(xxii) $\left(a^{\sharp}\right)^{k} a_{e, f}^{\dagger}=a_{e, f}^{\dagger}\left(a^{\sharp}\right)^{k}$;

(xxiii) $a^{k} a_{e, f}^{\dagger}=a_{e, f}^{\dagger} a^{k}$;

(xxiv) $a^{2 k-1}=a_{e, f}^{\dagger} a^{2 k+1} a_{e, f}^{\dagger}$;

$(\mathrm{xxv}) a\left(a_{e, f}^{\dagger}\right)^{k+1}=\left(a^{\sharp}\right)^{k}=\left(a_{e, f}^{\dagger}\right)^{k+1} a ;$

(xxvi) $a^{k} a a_{e, f}^{\dagger}+a_{e, f}^{\dagger} a a^{k}=2 a^{k}$;

$(\operatorname{xxvii})\left(a^{\sharp}\right)^{k+l-1}=\left(a_{e, f}^{\dagger}\right)^{l}\left(a^{\sharp}\right)^{k-1}=\left(a^{\sharp}\right)^{k-1}\left(a_{e, f}^{\dagger}\right)^{l}$;

(xxviii) $a a_{e, f}^{\dagger}\left(a^{\sharp}\right)^{k+l-1}=\left(a_{e, f}^{\dagger}\right)^{k}\left(a^{\sharp}\right)^{l} a=\left(a^{\sharp}\right)^{l} a\left(a_{e, f}^{\dagger}\right)^{k}$;

(xxix) $a$ is weighted EP with weights $f$ and $e$;

$(\mathrm{xxx}) a$ is both weighted EP with weights $e$ and $e$ and with weights $f$ and $f$;

(xxxi) $a^{k}$ is weighted EP with weights $e$ and $f$;

(xxxii) $a^{\sharp}$ is weighted EP with weights $e$ and $f$;

(xxxiii) $a a^{\sharp}=a a_{e, e}^{\dagger}=a a_{f, f}^{\dagger}\left(\right.$ or $\left.a a^{\sharp}=a_{e, e}^{\dagger} a=a_{f, f}^{\dagger} a\right)$;

(xxxiv) $a a^{\sharp}=a a_{e, f}^{\dagger}=a a_{f, e}^{\dagger}\left(\right.$ or $\left.a a^{\sharp}=a_{f, e}^{\dagger} a=a_{e, f}^{\dagger} a\right)$;

$(\mathrm{xxxv}) a a_{e, f}^{\dagger}\left(a^{k}+\lambda a_{e, f}^{\dagger}\right)=\left(a^{k}+\lambda a_{e, f}^{\dagger}\right) a a_{e, f}^{\dagger} ;$

(xxxvi) $a_{e, f}^{\dagger} a\left(a^{k}+\lambda a_{e, f}^{\dagger}\right)=\left(a^{k}+\lambda a_{e, f}^{\dagger}\right) a_{e, f}^{\dagger} a$;

(xxxvii) $\left(a+\lambda a_{e, e}^{\dagger}\right) A=\left(a+\lambda a_{f, f}^{\dagger}\right) A=\left(\lambda a+a^{3}\right) A$ and 
$\left(a+\lambda a_{e, e}^{\dagger}\right)^{-1}(0)=\left(a+\lambda a_{f, f}^{\dagger}\right)^{-1}(0)=\left(\lambda a+a^{3}\right)^{-1}(0)$

(xxxviii) $\left(a+\lambda a_{e, f}^{\dagger}\right) A=\left(\lambda a+a^{3}\right) A$ and $\left(a+\lambda a_{e, f}^{\dagger}\right)^{-1}(0)=\left(\lambda a+a^{3}\right)^{-1}(0)$, where $k, l \in \mathbb{N}$ and $\lambda \in \mathbb{C} \backslash\{0\}$.

Proof. Let $a \in A$ satisfying the hypothesis of the theorem. It is not difficult to prove that if $a$ is weighted $\mathrm{EP}$ with weights $e$ and $f$, then statements (i)(xiii) and (xix)-(xxxviii) hold. To prove the converse in this case, consider $L_{a}: A \rightarrow A$ and apply the left multiplication representation to the statements. According Theorem 2.8 and Remark 3.3(i), each statement is elaborated with $L_{a},\left(L_{a}\right)^{\sharp}$, and $\left(L_{a}\right)_{L_{e}, L_{f}}^{\dagger}$ instead of $a, a^{\sharp}$ and $a_{e, f}^{\dagger}$, respectively. Then apply Theorems 3.5 and 3.6 to prove that $L_{a} \in L(A)$ is weighted EP with weights $L_{e}$ and $L_{f}$ (recall that according to Lemma $2.4, L_{e}, L_{f} \in L(A)$ are invertible and positive). Finally, apply again Theorem 2.8 to prove that $a$ is weighted EP with weights $e$ and $f$.

To prove the equivalence between the condition of being weighted EP and statement (xiv), note that if $a a_{e, f}^{\dagger}=a_{e, f}^{\dagger} a$, then it is not difficult to prove that $a=\left(a^{2}+1-a_{e, f}^{\dagger} a\right) a_{e, f}^{\dagger}=a_{e, f}^{\dagger}\left(a^{2}+1-a a_{e, f}^{\dagger}\right)$ and $\left(a^{2}+1-a_{e, f}^{\dagger} a\right)^{-1}=$ $\left(a_{e, f}^{\dagger}\right)^{2}+1-a_{e, f}^{\dagger} a$. Clearly, statement (xiv) implies statement (xv), which in turn implies statement (xvi). On the other hand, statement (xvi) implies statement (vi).

Now consider statement (xvii). If $a_{e, f}^{\dagger}$ exists, then since the group inverse is unique, $a_{e, f}^{\dagger}=a^{\sharp}$. Then, according to [6, Lemma 1.4.5], statement (xvii) holds. If, on the other hand, $a \in A$ satisfies the condition of statement (xvii), then applying this condition to $b=a, a$ is weighted $\mathrm{EP}$ with weights $e$ and $f$.

Finally, to prove the equivalence between statements (i) and (xviii), suppose that $a_{e, f}^{\dagger}$ exists. Then as in the previous paragraph, $a_{e, f}^{\dagger}=a^{\sharp}$. Then, according to [12, Theorem 4.4], statement (xviii) holds. On the other hand, if $a_{e, f}^{\dagger}=f(a)$, where $f: U \rightarrow C$ is holomorphic $(\sigma(a) \subset U)$, then according to [5, Proposition 4.9, Chapter VII], $a$ is weighted $\mathrm{EP}$ with weights $e$ and $f$.

\section{References}

[1] E. Boasso, On the Moore-Penrose inverse, EP Banach space operators, and EP Banach algebra elements, J. Math. Anal. Appl. 339 (2008), no. 2, 1003-1014.

[2] — Factorizations of EP Banach space operators and EP Banach algebra elements, J. Math. Anal. Appl. 379 (2011), no. 1, 245-255.

[3] E. Boasso and V. Rakočević, Characterizations of EP and normal Banach algebra elements and Banach space operators, Linear Algebra Appl. 435 (2011), no. 2, 342-353.

[4] F. F. Bonsall and J. Duncan, Complete Normed Algebras, Springer Verlag, Berlin, Heidelberg, New York, 1973.

[5] J. B. Conway, A Course in Functional Analysis, Springer Verlag, New York, Berlin, Heidelberg, Tokio, 1985.

[6] D. S. Djordjević and V. Rakočević, Lectures on Generalized Inverses, Faculty of Sciences and Mathematics, University of Nis, Nis, 2008. 
[7] H. R. Dowson, Spectral Theory of Linear Operators, London, New York, San Francisco, 1978.

[8] L. T. Gardner, Square roots in Banach algebras, Proc. Amer. Math. Soc. 17 (1966), $132-134$.

[9] R. Harte and M. Mbekhta, On generalized inverses in $C^{*}$-algebras, Studia Math. 103 (1992), no. 1, 71-77.

[10] - On generalized inverses in $C^{*}$-algebras. II, Studia Math. 106 (1993), 129-138.

[11] C. King, A note on Drazin inverses, Pacific J. Math. 70 (1977), no. 2, 383-390.

[12] J. J. Koliha, A generalized Drazin inverse, Glasgow Math. J. 38 (1996), no. 3, 367-381.

[13] _ Elements of $C^{*}$-algebras commuting with their Moore-Penrose inverse, Studia Math. 139 (2000), no. 1, 81-90.

[14] J. J. Koliha, D. Djordjević, and D. Cvetković, Moore-Penrose inverse in rings with involution, Linear Algebra Appl. 426 (2007), no. 2-3, 371-381.

[15] G. Lumer, Semi-inner-product spaces, Trans. Amer. Math. Soc. 100 (1961), 29-43.

[16] M. Mbekhta, Partial isometries and generalized inverses, Acta Sci. Math. (Szeged) 70 (2004), no. 3-4, 767-781.

[17] D. Mosić and D. S. Djordjević, EP elements in Banach algebras, Banach J. Math. Anal. 5 (2011), no. 2, 25-32.

[18] Weighted-EP elements in $C^{*}$-algebras, Electron. J. Linear Algebra, accepted for publication.

[19] - Factorization of weighted-EP elements in $C^{*}$-algebas, Appl. Math. Comput., accepted for publication.

[20] T. Palmer, Unbounded normal operators on Banach spaces, Trans. Amer. Math. Soc. 133 (1968), 385-414.

[21] R. Penrose, A generalized inverse for matrices, Proc. Cambridge Philos. Soc. 51 (1955), 406-413.

[22] V. Rakočević, Moore-Penrose inverse in Banach algebras, Proc. Roy. Irish Acad. Sect. A 88 (1988), no. 1, 57-60.

[23] _ On the continuity of the Moore-Penrose inverse in Banach algebras, Facta Univ. Ser. Math. Inform. 6 (1991), 133-138.

[24] Y. Tian and H. Wang, Characterizations of EP matrices and weighted-EP matrices, Linear Algebra Appl. 434 (2011), no. 5, 1295-1318.

[25] I. Vidav, Eine metrische Kennzeichnung der selbstadjungierten Operatoren, Math. Z. 66 (1956), 121-128.

ENRICO BOASSO

Via Cristoforo Cancellieri 2

34137 Trieste - Ts, ITALy

E-mail address: enrico_odisseo@yahoo.it

Dragan S. DJordjeVić

Faculty of Sciences and Mathematics

UNIVERSITY OF Niš

P.O. Box 224, 18000, Niš, SERBiA

E-mail address: dragan@pmf.ni.ac.rs

Dijana Mosić

Faculty of Sciences and Mathematics

UNIVERSITY OF NIŠ

P.O. Box 224, 18000, Niš, SERBiA

E-mail address: dijana@pmf.ni.ac.rs 